\title{
Persistent left superior vena cava with an absent right superior vena cava in a 72-year-old male with multivessel coronary artery disease
}

\author{
K. Szymczyk ${ }^{1}$, M. Polguj², E. Szymczyk³ ${ }^{3}$ A. Majos ${ }^{1}$, P. Grzelak¹ ${ }^{1}$ L. Stefańczyk ${ }^{1}$ \\ ${ }^{1}$ Department of Radiology, Barlicki Memorial Teaching Hospital No. 1, Medical University of Lodz, Poland \\ 2Department of Angiology, Chair of Anatomy, Medical University of Lodz, Poland \\ ${ }^{3}$ Department of Cardiology, Medical University of Lodz, Poland
}

[Received 1 March 2013; Accepted 18 March 2013]

\begin{abstract}
Congenital anomalies of systemic veins are usually asymptomatic and found incidentally during ultrasonography, computed tomography (CT) or magnetic resonance examinations performed for other clinical indications. Persistent left superior vena cava (PLSVC) with absent right superior vena cava (RSVC) is the congenital aberration in the thoracic venous system which occurs in only $0.09 \%$ to $0.13 \%$ of patients who have congenital heart defects. In this paper, we present the extremely rare case of a 72-year-old male with PLSVC associated with an absence of RSVC, referred for coronary CT angiography. Multidetector CT angiography is a powerful tool for the detection of venous anomalies, which is essential before invasive procedures such as the implantation of pacemakers. (Folia Morphol 2013; 71, 3: 271-273)
\end{abstract}

Key words: systemic veins, congenital anomaly, computed tomography

\section{INTRODUCTION}

Anomalies of the superior vena cava (SVC) are the result of venous system variations occurring in the early embryonic period. The presence of persistent left superior vena cava (PLSVC) associated with the absence of a right superior vena cava (RSVC) is extremely rare. Congenital anomalies of systemic veins are usually asymptomatic and found incidentally during ultrasonography, computed tomography (CT) or magnetic resonance performed for other clinical indications. The wide availability of imaging techniques has allowed more frequent identification of vena cava anomalies, which should be correctly recognised by radiologists to enable the correct planning of surgical and interventional treatments e.g. pacemaker implantation.

\section{CASE REPORT}

A 72-year-old male with a history of arterial hypertension and chronic pulmonary obstructive disease, who was refusing to undergo cardiac catheterisation, was referred by cardiologist to the Department of Radiology for coronary CT angiography because of shortness of breath on exertion. A previously performed treadmill stress test was nondiagnostic and a transthoracic echocardiogram revealed the left ventricle to have good symmetric function with an ejection fraction of $58 \%$. Coronary CT angiography using a 64-slice scanner (Lightspeed VCT; General Electric; Wisconsin, USA) with retrospective ECG gating was performed. A smartprep series revealed the absence of the right-sided SVC and an abnormal structure with the flow of contrast media in venous phase on the left side of the aorta corresponding to that of PLSVC. Furt-

Address for correspondence: K. Szymczyk, Department of Radiology, Barlicki Memorial Teaching Hospital No. 1, Medical University of Lodz, ul. Kopcińskiego 22, 90-153 Łódź, Poland, tel/fax: +48 4267867 34, e-mail: konrad.oskar@gmail.com 
her scans confirmed this diagnosis and also showed critical stenosis in the left anterior descending artery and significant stenoses in the circumflex branch and right coronary artery. Enlarged mediastinal lymph nodes and a tumour in the $4^{\text {th }}$ segment of the right lung were additional findings. Two weeks later, venography $\mathrm{CT}$ of the chest was performed. For better visualisation of venous system, a contrast agent was injected intravenously in both forearms. Both right and left brachocephalic veins drained into the PLSVC, which carried blood to an enlarged coronary sinus (Figs. 1, 2). No right sided SVC was observed. The inferior vena cava terminated normally in the right atrium. In the presented case, no other anomalies of the heart or great vessels were detected.

\section{DISCUSSION}

Development of the SVC starts in the $4^{\text {th }}$ week of gestation, when it is derived from the right common cardinal vein and the right anterior cardinal vein. The RSVC is formed by the confluence of the left and right brachiocephalic veins and drains into the right atrium, while the left superior vena cava (LSVC) disappears during development [9]. According to Nsah et al. [8] this mechanism is due to compression of the left-sided vena cava between the left atrium and the hilum of the left lung. If the left anterior cardinal vein persists, it develops into a PLSVC and usually drains into the coronary sinus via the Marshall vein [18].

Superior vena cava anomalies are relatively uncommon [15]. PLSVC is observed in $0.3 \%$ of the general population and in $3-5 \%$ of patients with congenital heart disease $[3,5]$. However, in about $90 \%$ of these cases the right homologue persists [11]. Therefore, the presence of the LSVC associated with an absent right superior vena is the rarest condition, with a prevalence of $0.09-0.13 \%$ of patients who have congenital heart defects [17].

In 1850, Marshall [7] was the first to describe a PLSVC. However, it was only in 1966 that Roszel [14] detected this phenomenon in the living, by angiocardiography and by catheterisation. Contemporary, widely available imaging techniques including echocardiography, $\mathrm{CT}$, and magnetic resonance imaging have enabled the diagnosis of venous anomalies more frequently, which in the absence of congenital heart disease, usually remain asymptomatic. In the presented case, the multidetector $\mathrm{CT}$ venogram was crucial for the diagnosis. It should be mentioned that there are 2 protocols of performing CT venogram.

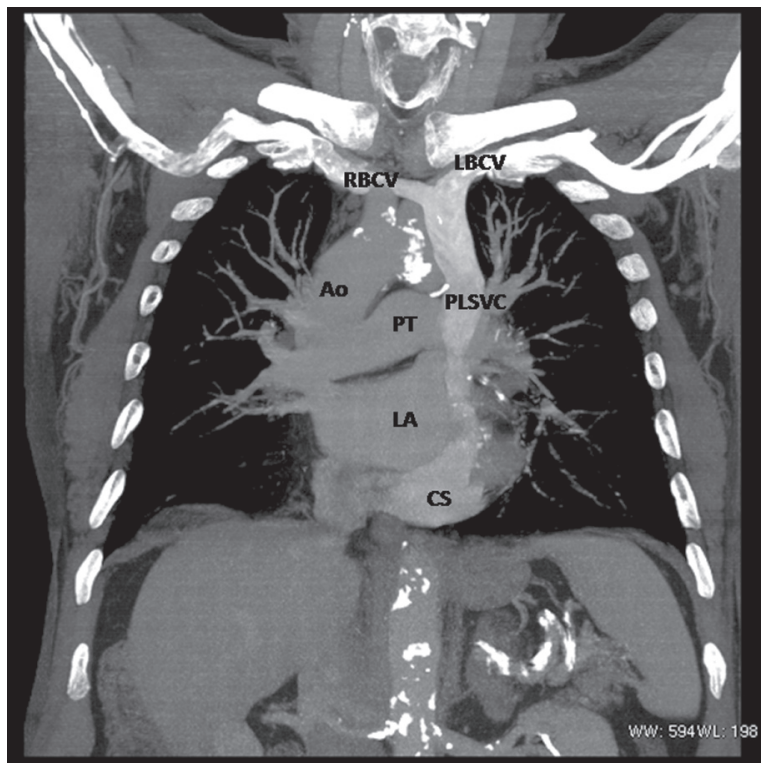

Figure 1. Computed tomography, maximum intensity projection, coronal plane; Ao — aorta; CS — coronary sinus; LA — left atrium; LBCV — left brachocephalic vein; PLSVC — persistent left superior vena cava; PT — pulmonary trunk; RBCV — right brachocephalic vein.

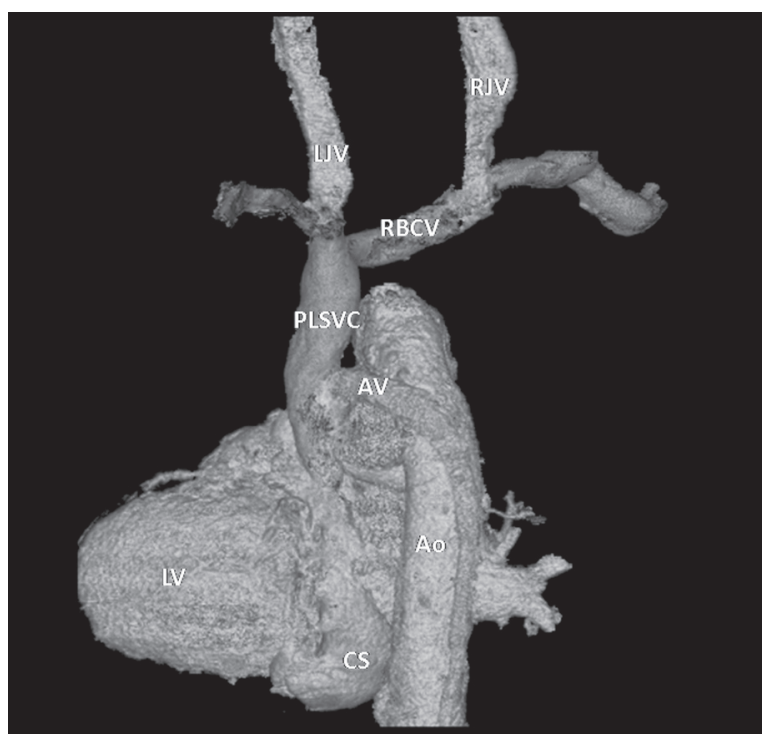

Figure 2. Computed tomography, volume rendering; $A_{0}$ - aorta; $\mathrm{AV}$ — azygos vein; CS — coronary sinus; LV — left ventricle; LJV — left jugular vein; PLSVC — persistent left superior vena cava; RBCV — right brachocephalic vein; RJV — right jugular vein.

The first, called a direct venogram, is based on acquisition during the first passage of a contrast agent through the veins, which requires an intravenous line to be inserted distally to the region of interest. The main disadvantage of this method results from artifacts caused by the 
inflow of undiluted contrast medium, especially in low flow vessels like veins. These artifacts are eliminated in the second protocol - the indirect venogram, in which acquisition takes place during reflow of contrast medium to the veins after passing through the pulmonary and circulatory system. However, the difficulty of predicting of the timing of acquisition and the insufficient opacity resulting from low contrast concentration has to be taken into consideration. In the presented case, a direct venogram was implemented with 2 venous lines placed into both forearms. This allows the exclusion of all venous connections that may not be detected with only 1 side contrast injection. A persistent LSVC may be also suspected during other tests: for example, by a chest $X$-ray which reveals a widened or extra shadow around the aortic knob, with a low-density line along the upper left cardiac margin, or when dilated coronary sinus is found on echocardiography [10].

In 2000, Giebel et al. [4] modified Starck's classification of anomalies of the SVC. Four variations were distinguished, based on embryonic development. In the first type, which is the most common, persistence of the RSVC and obliteration of the left occurs. The second type is typified by a PLSVC with same diameter as the RSVC. The third type is identified by a partial PLSVC - which is very thin. The fourth type, which is the rarest, is typified by persistence of the LSVC and obliteration of the right. According Giebel et al. [4] our case can be placed in the $4^{\text {th }}$ category.

Anomalies of the SVC should be taken into account during the insertion of central venous and pulmonary artery catheters or transvenous pacing leads when there is a longer than anticipated insertion length of a pulmonary artery catheter. Due to the abnormal path of the electrode, there can be a problem in obtaining a stable electrode position. Also, incidental access to the coronary sinus may cause hypotension, chest pain, perforation of the heart, tamponade and cardiac arrest $[1,6,13,16]$. Moreover, $\mathrm{P}$ wave abnormalities due to right atrial and ventricular enlargement, and arrhythmias due to stretching of the atrioventricular node and bundle of His may be observed on an ECG [19]. Also cardiac electrical instability affecting the sinus node may be present [2]. A persistent left vena cava is often associated with the coexistence of congenital heart disease e.g. ventricular and atrial septal defects which can potentially lead to paradoxical emboli and strokes $[12,16]$.

\section{CONCLUSIONS}

Multidetector CT angiography has proved its worth utility in depicting vascular anatomy due to its ability to visualise not only arteries but also veins, making this technique a valuable tool in the detection of venous anomalies. Appropriate identification of vascular anomalies is crucial before invasive procedures such us implantation of pacemakers. A precise knowledge of this anatomical variation is important, especially when central catheterisation via the subclavian or internal jugular vein proves difficult.

\section{REFERENCES}

1. Azocar RJ, Narang P, Talmor D, Lisbon A, Kaynar AM (2002) Persistent left superior vena cava identified after cannulation of the right subclavian vein. Anesth Analg, 95: 305-307.

2. Camm AJ, Dymond D, Spurrell RA (1979) Sinus node dysfunction associated with absence of right superior vena cava. Br Heart J, 41: 504.

3. Dibardino DJ, Fraser CD Jr, Dickerson HA, Heinle JS, McKenzie ED Kung G (2004) Left ventricular inflow obstruction associated with persistent left superior vena cava and dilated coronary sinus. J Thorac Cardiovasc Surg, 127: 959-962.

4. Giebel J, Fanghänel J, Hauser S, Paul I (2000) A case of a persistent left vena cava superior with atresia of the right atrial ostium of the coronary sinus. Ann Anat, 182: 191-194.

5. Gonzalez-Juanatey C, Testa A, Vidan J, Izquierdo R, Garcia-Castelo A, Daniel C, Armesto V (2004) Persistent left superior vena cava draining into the coronary sinus: report of 10 cases and literature review. Clin Cardiol, 27: 515-518.

6. Hindupur S, Lammoglia F (2006) Superior vena cava anomalies in the generation of angina pectoris. Cardiology, 105: 48-51.

7. Marshall J (1850) On the development of the great anterior veins in man and mammalia; including an account of certain remnants of foetal structure in the adult, a comparative view of these occasional peculiarities in the human subject. Phil Trans Royal Soc, 140: $133-170$.

8. Nsah E, Moore G, Hutchins G (1991) Pathogenesis of persistent left superior vena cava with a coronary sinus connection. Pediatr Pathol, 11: 261-269.

9. Ou P, Calcagni G, Marini D, Le Bidois J, Gallo P, Brunelle F, Bonnet $D$ (2007), Unusual systemic venous return with absence of superior caval veins. J Thorac Cardiovasc Surg, 133: 1368-1369.

10. Pahwa R, Kumar A (2003) Persistent left superior vena cava: an intensivist's experience and review of the literature. South Med J, 96: 528-529.

11. Peltier J, Destrieux C, Desme J, Renard C, Remond A, Velut S (2006) The persistent left superior vena cava: anatomical study, pathogenesis and clinical considerations. Surg Radiol Anat, 28: 206-210.

12. Postema PG, Rammeloo LA, van Litsenburg R, Rothuis EG, Hruda J (2008) Left superior vena cava in pediatric cardiology associated with extracardiac anomalies. Int J Cardiol, 123: 302-306.

13. Recupero A, Pugliatti P, Rizzo F, Carerj S, Cavalli G, de Gregorio C, D Bella G, Minutoli F, Arrigo F, Oreto G, Coglitore S (2007) Persistent left-sided superior vena cava: integrated noninvasive diagnosis. Echocardiography, 24: 982-986.

14. Roszel A (1966) Uber die Anomalie der Arteria subclavia dextra und die Persistenz der Vena cava superior sinistra. Anat Anz, 118: 348-367.

15. Sangam MR, Devi SS, Krupadanam K, Anasuya K (2011) Cor triloculare biventriculare with left superior vena cava. Folia Morphol, 70: 135-138.

16. Sarodia BD, Stoller JK (2000) Persistent left superior vena cava: case report and literature review. Respir Care, 45: 411-416.

17. Soward A, ten Cate F, Fioretti P, Roelandt J, Serruys PW (1986) An elusive persistent left superior vena cava draining into left atrium. Cardiology, 73: 368-371.

18. Tak T, Crouch E, Drake G (2002) Persistent left superior vena cava: incidence, significance and clinical correlates. Int J Cardiol, 2: 91-93.

19. Waikar H, Lahie Y, De Zoysa L, Chand P, Kamalanesan RP (2004) Systemic venous anomalies: absent right superior vena cava with persistent left superior vena cava. J Cardiothorac Vasc Anesth, 18: 332-335. 